\title{
Alterações na ictiocenose do rio Forqueta em função da instalação da Pequena Central Hidrelétrica Salto Forqueta, Putinga, Rio Grande do Sul
}

\author{
Alice Hirschmann ${ }^{1}$, Marco A. Majolo² \& Hamilton C. Z. Grillo ${ }^{3}$ \\ 1. PPG Biologia Animal, Laboratório de Ictiologia, UFRGS. Av. Bento Gonçalves, 9500, 91501-970 Porto Alegre, RS. \\ (alicehirschmann@yahoo.com.br) \\ 2. Autônomo. RS 115 Km 35, 95670-000 Gramado, RS. (mmajolo@hotmail.com) \\ 3. Museu de Ciências Naturais, Centro Universitário UNIVATES. Rua Avelino Tallini, 171, Caixa Postal 155, Bairro Universitário, \\ 95900-000 Lajeado, RS. (hgrillo@univates.br)
}

\begin{abstract}
Alterations on the fish assemblage of the Forqueta River due the installation of the Small Hydroelectrical Power-Station Salto Forqueta, Putinga, Rio Grande do Sul, Brazil. This study aimed to verify the alterations on the fish assemblage due the installation of the Small Hydroelectrical Power-Station Salto Forqueta in the course of the Forqueta River. The study area is located in the Taquari River basin, between the municipalities of Putinga (left margin of the river) and São José do Herval (right margin of the river). The study encompassed four phases: Phase I - from dec/2000 to feb/2002 (before damming), Phase II - from dec/ 2002 to jan/2004 (first year following damming), Phase III - from may/2004 to apr/2005 (second year following damming) and Phase IV - from may/2005 to jul/2006 (third year following damming). The samples were taken using fishing nets with diverse mashes. It was registered 28 species during Phase I, 21 species during Phase II, 19 species during Phase III and 18 species during Phase IV. The abundance rates, during the four phases, varied among the following species: Astyanax spp., Hemiancistrus punctulatus Cardoso \& Malabarba, 1999, Steindachnerina biornata (Pearson, 1924), Oligosarcus jenynsii (Günther, 1864) and Geophagus brasiliensis (Quoy \& Gaimard, 1824). It was verified a range of alterations in the local fish assemblage, nevertheless, these alterations were not statistically significant.
\end{abstract}

KEYWORDS. Ichthyofauna, reservoir, Taquari Valley, Hydroelectrical Power-Station.

RESUMO. Este estudo teve como objetivo verificar alterações na taxocenose de peixes devido à instalação da Pequena Central Hidrelétrica Salto Forqueta no curso do rio Forqueta. A área de estudo localiza-se na bacia hidrográfica do rio Taquari, entre os municípios de Putinga (margem esquerda do rio) e São José do Herval (margem direita do rio). O estudo abrangeu quatro etapas: Etapa I - dez/2000 a fev/2002 (anterior ao represamento), Etapa II - dez/2002 a jan/2004 (primeiro ano subseqüente ao represamento), Etapa III - maio/ 2004 a abr/2005 (segundo ano após o represamento) e Etapa IV - maio/2005 a jul/2006 (terceiro ano após o represamento). As amostragens foram realizadas com redes de espera de malhas diversas. Foram registradas 28 espécies na Etapa I, 21 espécies na Etapa II, 19 espécies na Etapa III e 18 espécies na Etapa IV, sendo que as maiores taxas de abundância nas quatro etapas de monitoramento variaram entre as espécies Astyanax spp., Hemiancistrus punctulatus Cardoso \& Malabarba, 1999, Steindachnerina biornata (Pearson, 1924), Oligosarcus jenynsii (Günther, 1864) e Geophagus brasiliensis (Quoy \& Gaimard, 1824). Verificaram-se diversas alterações na ictiocenose local, porém, as alterações não se mostraram estatisticamente significativas.

PALAVRAS CHAVE. Ictiofauna, reservatório, Vale do Taquari, PCH.

A composição ictiofaunística ao longo dos rios acompanha as mudanças ambientais no tempo e no espaço, com a efetividade de suas barreiras naturais (representadas por corredeiras e cachoeiras) e com o tamanho e multiplicidade de nichos ecológicos. Os rios são sistemas abertos que participam de todos processos ecológicos que ocorrem nas bacias hidrográficas (BARRElla et al., 2000), influenciando fortemente na composição da biota.

A inclusão de barreiras artificiais em ecossistemas aquáticos continentais podem desencadear inúmeros processos com repercussões ecológicas, econômicas e sociais, em uma escala global, regional ou local. Tais ecossistemas artificiais podem ocasionar alteração do fluxo dos rios, do transporte de nutrientes e sedimentos, interferência na migração e reprodução da ictiofauna, produzindo uma completa reorganização dos sistemas. Porém, podem proporcionar benefícios econômicos e sociais, tais como a reserva de água para hidroeletricidade, irrigação, navegação e água para abastecimento público (TuNDISI, 1999).
As Pequenas Centrais Hidrelétricas ( $\mathrm{PCH})$ são usinas de pequeno porte, com potência instalada de até 30 MW. A PCH Salto Forqueta apresenta potência de 6,5 MW, seu reservatório abrange 34,6 ha com um volume de $2.260 .000 \mathrm{~m}^{3}$, sendo que 12,8 ha representam o leito original do rio e 21,8 ha correspondiam às áreas cobertas por vegetação nativa localizadas na sua margem. De acordo com TEIXEIRA et al. (1986) e JASPER et al. (2005), a PCH Salto Forqueta atingiu uma região que apresentava originalmente uma riquíssima cobertura vegetal, com uma composição fitoecológica ímpar, inserida em uma área de tensão ecológica entre as formações Floresta Estacional Decidual e Floresta Ombrófila Mista.

É esperado que o impacto causado pelos represamentos possa provocar a depleção ou mesmo a extinção local de algumas populações, sendo que as mais atingidas são aquelas tipicamente reofílicas, que têm seus locais de desova e criadouros naturais reduzidos ou mesmo eliminados (Agostinho, 1992). Por outro lado, podem favorecer outras espécies, aumentando as suas chances de sobrevivência e reprodução (BARRELLA et al., 2000). 
A instabilidade do novo ambiente, fruto não apenas do impacto inicial do represamento, mas também de perturbações não cíclicas produzidas pela operação da barragem ou por outras ações antropogênicas, torna as comunidades instáveis e gradativamente mais simples. Desta forma faz-se necessário verificar como os indicadores ecológicos (abundância, constância de ocorrência, riqueza de espécies, diversidade, equitabilidade) variam após a intervenção (SCHINDLER, 1987).

O objetivo deste estudo foi verificar possíveis alterações na estrutura e composição da assembléia de peixes após a implantação da PCH Salto Forqueta no rio Forqueta.

\section{MATERIAL E MÉTODOS}

A área de estudo localiza-se na bacia hidrográfica do rio Taquari, entre os municípios de Putinga/RS e São José do Herval, Rio Grande do Sul, $29^{\circ} 04^{\prime} \mathrm{S}$ e $52^{\circ} 12^{\prime} \mathrm{W}$ (Fig. 1). Neste ponto, o rio Forqueta apresenta-se encaixado em um vale de encostas muito íngrimes, alternando poços e corredeiras, eventualmente intercalados por saltos de pequeno porte. $\mathrm{O}$ rio Forqueta tem sua foz no rio Taquari, entre os municípios de Lajeado e Arroio do Meio, sendo que a área de estudo situa-se a aproximadamente $45 \mathrm{Km}$ a montante desta confluência.

O estudo abrangeu quatro etapas: Etapa I - dez/ 2000 a fev/2002 (anterior ao represamento), Etapa II dez/2002 a jan/2004 (primeiro ano subseqüente ao represamento), Etapa III - maio/2004 a abr/2005 (segundo ano após o represamento) e Etapa IV - maio/2005 a jul/ 2006 (terceiro ano após o represamento).

$\mathrm{Na}$ Etapa I, foram definidos quatro pontos de coleta, sendo dois localizados a montante do local do barramento e dois a jusante do mesmo, com distância aproximada de 1,5 $\mathrm{Km}$. Foram realizadas 12 amostragens com nove redes de espera de malhas $1,5 \mathrm{~cm}, 2,5 \mathrm{~cm}$ e 3,5 cm entre nós adjacentes com $20 \mathrm{~m}$ de comprimento e 1,40 m de altura, dispostas em ambas as margens. As redes permaneceram armadas durante um período de 24 horas, sendo revisadas ao anoitecer, ao amanhecer e ao meio do dia, momento da retirada.

Para as Etapas II, III e IV, foram escolhidos apenas dois dos pontos de coleta previamente amostrados na Etapa I, sendo um a montante e um a jusante do

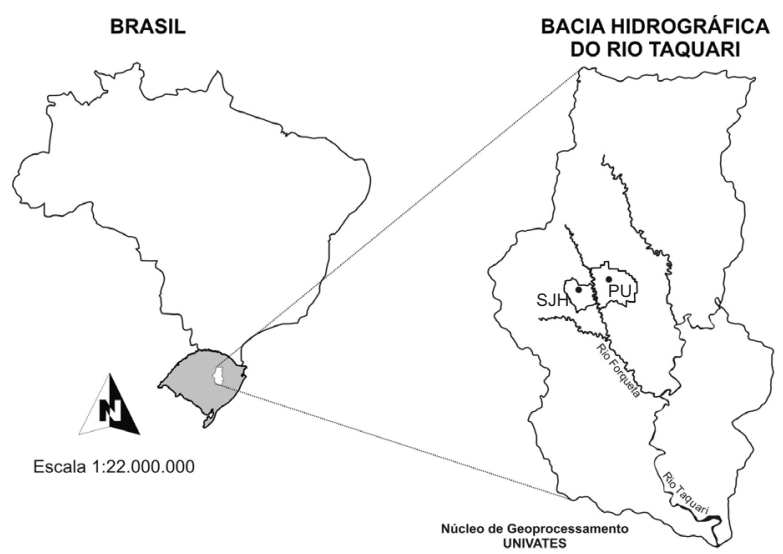

Fig. 1. Localização da área de estudo no rio Forqueta, entre os municípios de Putinga (PU) e São José do Herval (SJH), Rio Grande do Sul. barramento. No reservatório foram empregadas seis redes de espera de malhas $1,5 \mathrm{~cm}, 2,5 \mathrm{~cm}$ e $3,5 \mathrm{~cm}$ entre nós adjacentes, com $50 \mathrm{~m}$ de comprimento e 1,40 $\mathrm{m}$ de altura e dispostas como na Etapa I. No ponto a jusante a metodologia de coleta seguiu conforme a Etapa I. Foram realizadas seis amostragens nas Etapas II e IV e sete amostragens na Etapa III.

Adicionalmente, nas corredeiras e baixios, durante a Etapa I, foi empregado puçá de tela plástica ( 1 m x 0,4 $\mathrm{m})$. Os dados obtidos por este método foram apenas qualitativos, não incluídos nas análises ecológicas e estatísticas.

Os indivíduos coletados foram fixados em formol $10 \%$ e, em laboratório, foram conservados em álcool $70 \%$. Para identificação das espécies foram consultados especialistas e bibliografia, tal como MALABARBA (1989), Koch et al. (2000), NAKATANi et al. (2001) e ReIs et al. (2003). O material de estudo encontra-se depositado na coleção zoológica científica do Museu de Ciências Naturais da UNIVATES.

Para análise estatística, todos os dados quantitativos tiveram o esforço de amostragem padronizado para $252 \mathrm{~m}^{2}$ de rede/h, esforço empregado no ponto de amostragem a montante do barramento, pósenchimento.

Para visualização comparativa entre os dados antes e depois do barramento, construiu-se um gráfico representando as alterações na abundância das espécies. Para tal, fez-se a média da abundância absoluta de cada uma das espécies nas três etapas subseqüentes ao barramento. Tais médias foram comparadas à abundância absoluta das espécies na Etapa I, onde ambos dados foram transformados $(\log n+1)$. Também foram feitas curvas de abundância, onde a abundância expressa em $\log \mathrm{n}+1$ é plotada contra o rank de espécies em ordem decrescente de abundância.

Com o intuito de caracterizar a ictiocenose, foram empregados o Índice de Shannon (H'), a Equitabilidade de Pielou (J = H'/H'max) e a Riqueza de espécies de Margalef, através do programa Divers, e o Índice de Constância de Dajoz (1983), C= (p x 100)/P, onde p = número de amostras que a espécie ocorre, $\mathrm{P}=$ número total de amostragens efetuadas e $\mathrm{C}=$ índice de constância. De acordo com os valores de $\mathrm{C}$ as espécies foram agrupadas nas seguintes categorias: espécies constantes - presentes em mais de $50 \%$ das amostragens; espécies acessórias - presentes em $25 \%$ a $50 \%$ das amostragens; espécies acidentais - presentes em menos de $25 \%$ das amostragens. Também foram detectadas as espécies raras para cada etapa do monitoramento, isto é, que somente foram capturadas uma ou duas vezes durante o período. O teste $\mathrm{t}$ de Student foi realizado através do programa Divers e a análise de variância (ANOVA) foi realizada através do programa GraphPad InStat 3. A análise de cluster com índice de similaridade de Bray-Curtis referente aos trechos montante e jusante das quatro etapas do monitoramento foi realizada através do programa Past.

\section{RESULTADOS}

O levantamento ictiofaunístico referente à Etapa I, resultou na captura de 2.721 indivíduos pertencentes a 
28 espécies, distribuídas em 11 famílias e quatro ordens. A ordem Characiformes apresentou 12 espécies $(42,86 \%)$ e a ordem Siluriformes 13 espécies $(46,43 \%)$. Na Etapa II, foram capturados 1.353 indivíduos de 21 espécies, pertencentes a oito famílias e distribuídas em quatro ordens, sendo que as mais representativas foram a ordem Characiformes, com oito espécies $(38,10 \%)$ e a ordem Siluriformes, com sete espécies $(33,33 \%)$. Na Etapa III, capturou-se 1.466 indivíduos de 19 espécies pertencentes a sete famílias e três ordens. Foram obtidas nove espécies de Characiformes $(47,37 \%)$ e seis espécies de Siluriformes $(31,58 \%)$. Finalmente, na Etapa IV foram capturados 1.335 indivíduos de 18 espécies pertencentes a oito famílias e cinco ordens, sendo registradas sete espécies da ordem Characiformes $(38,89 \%)$ e cinco espécies da ordem Siluriformes $(27,78 \%)$.

Adicionalmente, com a utilização do puçá, registrou-se a ocorrência de quatro outras espécies na Etapa I (Eurycheilichthys sp., Hisonotus sp., Heptapterus mustelinus (Valenciennes, 1835) e Synbranchus marmoratus Bloch, 1795). O registro da espécie Corydoras paleatus (Jenyns, 1842) na Etapa I foi obtido somente através da análise de conteúdo estomacal de um indivíduo de Oligosarcus jenynsii (Günther, 1864). Obteve-se o registro total de 36 espécies de peixes para a área de estudo no rio Forqueta.

A relação das espécies registradas em cada etapa está apresentada na tabela I. Pode-se observar que, apesar do registro de mais três espécies ao longo das Etapas II, III e IV, das espécies registradas antes do barramento, oito não foram registradas na Etapa II e 11 não foram registradas nas Etapas III e IV.

De acordo com o índice de constância de ocorrência, das 28 espécies registradas durante a Etapa I, 12 foram consideradas constantes, cinco acessórias e 11 acidentais. Quanto à Etapa II, das 21 espécies registradas, 14 foram consideradas constantes, três acessórias e quatro acidentais. Na Etapa III, das 19 espécies registradas, 12 foram consideradas constantes, cinco acessórias e duas acidentais. Por fim, na Etapa IV, das 18 espécies registradas, 11 foram consideradas constantes, três acessórias e quatro acidentais (Tab. I).

Ao longo do monitoramento observou-se que 17 espécies apresentaram pouca ou nenhuma variação em sua constância de ocorrência. Por outro lado, nove espécies não foram registradas após o represamento do rio: Astyanax fasciatus (Cuvier, 1819), Hypobrycon sp., Characidium pterostictum Gomes, 1947, Cyphocharax voga (Hensel, 1870), Ancistrus brevipinnis (Regan, 1904), Hypostomus aspilogaster (Cope, 1894), Rineloricaria strigilata (Hensel, 1868), Pimelodus maculatus La Cepède, 1803 e Eigenmannia trilineata Lópes \& Castello, 1966 (Tab. I).

Outras variações foram observadas quanto à constância com que se apresentaram algumas espécies. As espécies Rineloricaria cadeae (Hensel, 1868), Glanidium melanopterum Miranda Ribeiro, 1918 e Crenicichla punctata Hensel, 1870, que se apresentavam constantes no período anterior ao barramento, tiveram suas constâncias de ocorrência visivelmente diminuídas. Por outro lado, Astyanax jacuhiensis Cope, 1894, Hyphessobrycon luetkenii (Boulenger,1887) e Geophagus brasiliensis (Quoy \& Gaimard, 1824) apresentaram um aumento considerável, tanto na constância de ocorrência quanto na abundância (Tab. I e Fig. 2).
Assim, verificou-se que a maioria das espécies consideradas acidentais e raras na Etapa I, não foram mais registradas após o barramento do rio, e outras espécies consideradas constantes sofreram alterações principalmente na abundância, com a maioria mantendo o status constante nas amostragens (Tab. I e Fig. 2).

As espécies de Astyanax spp. (40,28\%), Hemiancistrus punctulatus Cardoso \& Malabarba, 1999 $(24,55 \%)$ e Steindachnerina biornata (Pearson, 1924) $(10,88 \%)$ foram as mais abundantes no rio Forqueta durante a Etapa I. Na Etapa II, as espécies que apresentaram maior abundância foram Astyanax spp. $(27,17 \%), H$. punctulatus $(24,82 \%)$, S. biornata $(13,32 \%)$ e O. jenynsii $(8,69 \%)$. Já na Etapa III, as maiores abundâncias foram observadas para as espécies, $S$. biornata $(41,40 \%)$, Astyanax spp. (27,37\%), O. jenynsii $(9,99 \%)$ e G. brasiliensis (7,33 \%). Na Etapa IV, as espécies mais abundantes foram Astyanax spp. $(25,88 \%), S$. biornata $(21,88 \%)$, H. luetkenii $(16,70 \%)$, G. brasiliensis $(14,05 \%)$ e $O$. jenynsii $(10,81 \%)$. Com relação as espécies mais abundantes na área, verificou-se que a representatividade de Astyanax spp. diminuiu nas etapas subseqüentes ao barramento, embora tenha se mantido entre as espécies mais abundantes. Em contrapartida, $H$. punctulatus, uma das espécies mais abundantes nas primeiras duas etapas, apresentou uma drástica redução em seus números nas Etapas III e IV. Quanto às espécies $S$. biornata, $O$. jenynsii e $H$. luetkenii, observou-se um gradativo aumento na abundância ao longo das etapas. Quanto à G. brasiliensis, espécie rara nas duas primeiras etapas, sua representatividade aumentou de $0,04 \%$ (Etapa I) para $14,05 \%$ (Etapa IV), sendo a quarta espécie mais abundante (Fig. 2).

A ordem Characiformes foi a mais abundante em todas as etapas (I: $59,61 \%$; II: $57,37 \%$; III: $86,6 \%$ e IV: $80,56 \%$ ), apresentando aumento após o barramento. Já a ordem Siluriformes apresentou um grande decréscimo em sua abundância (I: 34,85\%; II: 37,41\%; III: 4,07\% e IV: $2,39 \%)$, apresentando taxas menores que a ordem Perciformes a partir da Etapa III (I: 5,41\%; II: 5,17\%; III: 9,33\% e IV: $17,57 \%)$.

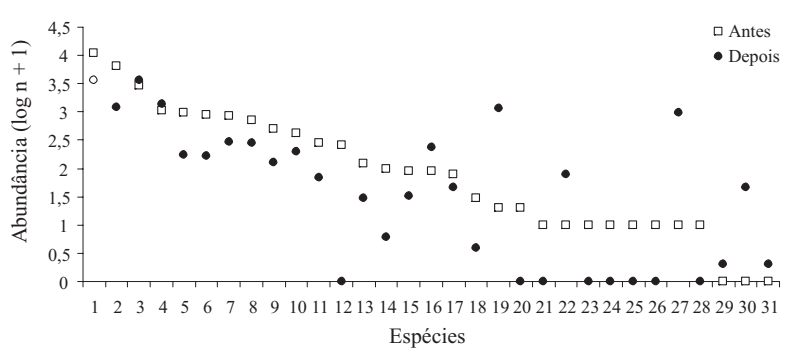

Fig. 2. Gráfico comparativo da abundância das espécies no rio Forqueta, área de abrangência da PCH Salto Forqueta, entre os municípios de Putinga e São José do Herval, Rio Grande do Sul, antes e depois do barramento, entre dezembro de 2000 e julho de 2006. 1: Astyanax spp.; 2: H. punctulatus; 3: S. biornata; 4: O. jenynsii; 5: B. iheringii; 6: R. cadeae; 7: G. labiatus; 8: Rhamdia sp.; 9: C. punctata; 10: H. commersoni; 11: G. melanopterum; 12: H. aspilogaster; 13: A. brevipinnis; 14: Australoheros sp.; 15: C. alburnus; 16: H. malabaricus; 17: R. eriarcha; 18: G. carapo; 19: H. luetkenii; 20: C. voga; 21: A. fasciatus; 22: A. jacuhiensis; 23: Hypobrycon sp.; 24: C. pterostictum; 25: R. strigilata; 26: P. maculatus; 27: G. brasiliensis; 28: E. trilineata; 29: D. speculiferum; 30: G. gymnogenys; 31: C. carpio. 
Tabela I. Lista das espécies de peixes amostradas no rio Forqueta, área de abrangência da PCH Salto Forqueta, entre os municípios de Putinga e São José do Herval, Rio Grande do Sul, entre dezembro de 2000 e julho de 2006, e suas respectivas constâncias de ocorrência durante as quatro etapas do estudo (*, espécie encontrada em análise de conteúdo estomacal de Oligosarcus jenynsii; **, espécies raras; ***, espécies capturadas apenas com puçá, desconsideradas na análise de constância de ocorrência).

\begin{tabular}{|c|c|c|c|c|}
\hline \multirow{2}{*}{ Lista de Espécies } & \multicolumn{4}{|c|}{ Constância de Ocorrência } \\
\hline & Etapa I & Etapa II & Etapa III & Etapa IV \\
\hline \multicolumn{5}{|l|}{ CHARACIFORMES } \\
\hline \multicolumn{5}{|l|}{ CHARACIDAE } \\
\hline Astyanax fasciatus (Cuvier, 1819) & Acidental** & - & - & - \\
\hline Astyanax jacuhiensis Cope, 1894 & Acidental ${ }^{* *}$ & Acessória & Constante & Constante \\
\hline Astyanax spp. & Constante & Constante & Constante & Constante \\
\hline Bryconamericus iheringii (Boulenger,1887) & Constante & Constante & Acessória** & Constante \\
\hline Diapoma speculiferum Cope, 1894 & - & - & Acidental** & - \\
\hline Hyphessobrycon luetkenii (Boulenger,1887) & Acidental ${ }^{* *}$ & Constante & Constante & Constante \\
\hline Hypobrycon sp. & Acidental** & - & - & - \\
\hline Oligosarcus jenynsii (Günther, 1864) & Constante & Constante & Constante & Constante \\
\hline \multicolumn{5}{|l|}{ CRENICHIDAE } \\
\hline Characidium pterostictum Gomes, 1947 & Acidental ${ }^{* *}$ & - & - & - \\
\hline \multicolumn{5}{|l|}{ CURIMATIDAE } \\
\hline Steindachnerina biornata (Pearson, 1924) & Constante & Constante & Constante & Constante \\
\hline Cyphocharax voga (Hensel, 1870) & Acidental ${ }^{* *}$ & - & - & - \\
\hline \multicolumn{5}{|l|}{ ERYTHRINIDAE } \\
\hline Hoplias malabaricus (Bloch, 1794) & Acessória & Constante & Constante & Constante \\
\hline \multicolumn{5}{|l|}{ SILURIFORMES } \\
\hline \multicolumn{5}{|l|}{ LORICARIIDAE } \\
\hline Ancistrus brevipinnis (Regan, 1904) & Constante & Acessória & - & - \\
\hline Eurycheilichthys sp.*** & - & - & - & - \\
\hline Hemiancistrus punctulatus Cardoso \& Malabarba, 1999 & Constante & Constante & Constante & Acessória \\
\hline Hisonotus sp.*** & - & - & - & - \\
\hline Hypostomus aspilogaster (Cope, 1894) & Acessória & - & - & - \\
\hline Hypostomus commersoni Valenciennes, 1836 & Constante & Constante & Constante & Constante \\
\hline Rineloricaria cadeae (Hensel, 1868) & Constante & Constante & Acessória** & Acidental** \\
\hline Rineloricaria strigilata (Hensel, 1868) & Acidental $* *$ & - & - & - \\
\hline \multicolumn{5}{|l|}{ CALLICHTHYDAE } \\
\hline Corydoras paleatus (Jenyns, 1842)* & - & - & - & - \\
\hline \multicolumn{5}{|l|}{ PIMELODIDAE } \\
\hline Pimelodus maculatus La Cepède, 1803 & Acidental** & - & - & - \\
\hline \multicolumn{5}{|l|}{ HEPTAPTERIDAE } \\
\hline Rhamdia sp. & Constante & Constante & Constante & Constante \\
\hline Rhamdella eriarcha (Eigenmann \& Eigenmann, 1888) & Acessória & Constante & Acessória & Acessória \\
\hline Heptapterus mustelinus (Valenciennes, 1835)*** & - & - & - & - \\
\hline \multicolumn{5}{|l|}{ AUCHENIPTERIDAE } \\
\hline Glanidium melanopterum Miranda Ribeiro, 1918 & Constante & Constante & Acidental $* *$ & - \\
\hline \multicolumn{5}{|l|}{ PERCIFORMES } \\
\hline \multicolumn{5}{|l|}{ CICHLIDAE } \\
\hline Gymnogeophagus labiatus (Hensel, 1870) & Constante & Constante & Constante & Constante \\
\hline Gymnogeophagus gymnogenys (Hensel, 1870) & - & Acidental & Acessória & - \\
\hline Geophagus brasiliensis (Quoy \& Gaimard, 1824) & Acidental ${ }^{* *}$ & Acidental** & Constante & Constante \\
\hline Australoheros sp. & Acessória & Acidental** & - & Acessória** \\
\hline Crenicichla punctata Hensel, 1870 & Constante & Constante & Constante & Acidental \\
\hline \multicolumn{5}{|l|}{ GYMNOTIFORMES } \\
\hline \multicolumn{5}{|l|}{ GYMNOTIDAE } \\
\hline Gymnotus carapo Linnaeus, 1758 & Acidental & Acidental ${ }^{* *}$ & - & Acidental** \\
\hline \multicolumn{5}{|l|}{ STERNOPYGIDAE } \\
\hline Eigenmannia trilineata Lópes \& Castello, 1966 & Acidental** & - & - & - \\
\hline SYNBRANCHIFORMES & & & & \\
\hline SYNBRANCHIDAE & & & & \\
\hline Synbranchus marmoratus Bloch, 1795 & - & - & - & - \\
\hline CYPRINIFORMES & & & & \\
\hline CYPRINIDAE & & & & \\
\hline Cyprinus carpio Linnaeus, 1758 & - & - & - & Acidental** \\
\hline
\end{tabular}


As alterações na abundância das espécies refletem modificações no padrão de distribuição das abundâncias na assembléia, que se torna gradativamente mais simples, com a perda de espécies ao longo das etapas de monitoramento (Fig. 3).

No que se refere às comparações das abundâncias das espécies entre montante e jusante, em cada uma das etapas, não foram encontradas diferenças significativas, como pode ser observado na tabela II.

Com relação aos índices ecológicos aplicados (Tab. II), verificou-se um aumento da diversidade na Etapa II em relação à Etapa I e um aumento da equitabilidade, com uma posterior redução na Etapa III. A Etapa I apresentou os índices mais elevados de riqueza de espécies. Na Etapa IV houve um modesto aumento na diversidade e equitabilidade em relação à Etapa III, embora menores do que as observadas na Etapa II.

O resultado do teste $t$ de Student (Tab. II) mostrou que a Etapa II foi a única a apresentar diferença estatisticamente significativa entre montante e jusante quanto a diversidade de espécies $(\mathrm{t}=6,107 ; \mathrm{p}=0,0001)$.

A análise de cluster com índice de similaridade de Bray-Curtis, para os trechos a montante e jusante, entre

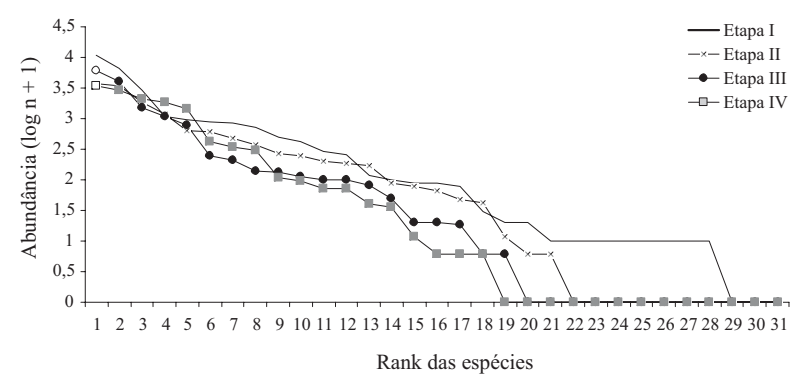

Fig. 3. Curvas de abundância das espécies para as quatro etapas do estudo no rio Forqueta, área de abrangência da PCH Salto Forqueta, entre os municípios de Putinga e São José do Herval, Rio Grande do Sul entre dezembro de 2000 e julho de 2006. as quatro etapas de monitoramento, identificou dois clusters, formados respectivamente pelas Etapas I e II e pelas Etapas III e IV, similares entre si pouco mais de $50 \%$. (Fig. 4). Observa-se uma grande similaridade (80\%) entre montante e jusante na Etapa I (anterior ao represamento) e uma subseqüente diminuição na similaridade entre os trechos montante e jusante ao longo das etapas do estudo, revelando o maior distanciamento dos mesmos na Etapa IV.

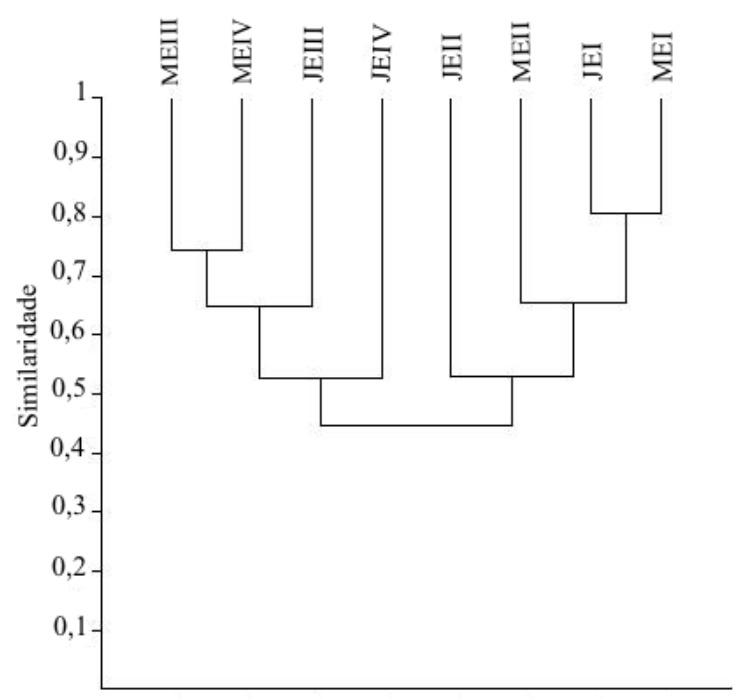

Fig. 4. Dendograma relativo à análise de cluster comparativa entre os trechos a montante e a jusante nas quatro etapas de monitoramento da ictiofauna no rio Forqueta, área de abrangência da PCH Salto Forqueta, entre os municípios de Putinga e São José do Herval, Rio Grande do Sul, entre dezembro de 2000 e julho de 2006. MEI, Montante Etapa I; JEI, Jusante Etapa I; MEII, Montante Etapa II; JEII, Jusante Etapa II; MEIII, Montante Etapa III; JEIII, Jusante Etapa III; MEIV, Montante Etapa IV e JEIV, Jusante Etapa IV.

Tabela II. Representação dos índices de diversidade de Shannon (H'), equitabilidade (E) e riqueza de espécies de Margalef (R), juntamente com a análise de variância $(\mathrm{F}$ e $\mathrm{P})$ e teste $\mathrm{t}$ de Student $(\mathrm{t})$ referentes à ictiofauna do rio Forqueta durante as quatro etapas de monitoramento na área de abrangência da PCH Salto Forqueta, entre os municípios de Putinga e São José do Herval, Rio Grande do Sul, entre dezembro de 2000 e julho de 2006. EI, Etapa I; EII, Etapa II e EIII, Etapa III.

\begin{tabular}{|c|c|c|c|c|c|c|c|}
\hline Etapas & $\mathrm{H}^{\prime}$ & $\mathrm{E}$ & $\mathrm{R}$ & $\mathrm{F}$ & $\mathrm{P}$ & $\mathrm{t}$ & $\mathrm{P}$ \\
\hline Montante-EI & 1,82518 & 0,60926 & 2,58667 & \multirow[t]{2}{*}{ - } & \multirow[t]{2}{*}{-} & \multirow{2}{*}{1,21888} & \multirow[t]{2}{*}{0,11150} \\
\hline Jusante-EI & 1,88203 & 0,60023 & 3,11330 & & & & \\
\hline Montante-EII & 2,17910 & 0,71574 & 2,93966 & \multirow{2}{*}{-} & \multirow{2}{*}{-} & \multirow{2}{*}{6,10725} & \multirow{2}{*}{0,00001} \\
\hline Jusante-EII & 1,81779 & 0,67126 & 2,28912 & & & & \\
\hline Montante-EIII & 1,63004 & 0,58791 & 2,17496 & \multirow{2}{*}{ - } & \multirow{2}{*}{-} & \multirow{2}{*}{0,71953} & \multirow{2}{*}{0,23600} \\
\hline Jusante-EIII & 1,67549 & 0,61871 & 2,26996 & & & & \\
\hline Montante-EIV & 1,81514 & 0,64067 & 2,37621 & \multirow{2}{*}{-} & \multirow{2}{*}{-} & \multirow{2}{*}{1,04080} & \multirow{2}{*}{0,29818} \\
\hline Jusante-EIV & 1,76310 & 0,73527 & 1,61067 & & & & \\
\hline Montante-EI, II, III e IV & - & - & - & 0,994 & 0,39 & - & - \\
\hline Jusante-EI, II, III e IV & - & - & - & 1,976 & 0,12 & - & - \\
\hline $\begin{array}{l}\text { Montante e Jusante- } \\
\text { EI, II, III e IV }\end{array}$ & - & - & - & 1,488 & 0,17 & - & - \\
\hline Etapa I & 1,88179 & 0,56473 & 3,41394 & \multirow{4}{*}{0,564} & \multirow{4}{*}{0,68} & & \\
\hline Etapa II & 2,14419 & 0,70428 & 2,77361 & & & & \\
\hline Etapa III & 1,69958 & 0,57722 & 2,46904 & & & & \\
\hline Etapa IV & 1,95933 & 0,67788 & 2,36171 & & & & \\
\hline
\end{tabular}




\section{DISCUSSÃO}

O conhecimento sobre a ictiofauna da bacia do rio Taquari é parco, estando disponível apenas em poucos trabalhos não publicados (dissertação de mestrado e resumos). A única literatura publicada refere-se ao rio das Antas (Agostinho et al., 2003), que é considerado a parte superior do rio Taquari, formando a grande bacia hidrográfica Taquari-Antas. Nesse trabalho é relatada a presença de 54 espécies de peixes, das quais 17 não foram registradas no presente estudo. Por ouro lado, seis das 36 espécies registradas no rio Forqueta não foram registradas no trabalho de AgostinHo et al. (2003). Tais diferenças podem estar associadas ao ambiente e aos métodos de amostragem utilizados por estes estudos, que não os torna diretamente comparáveis.

A composição da assembléia de peixes observada no presente estudo segue o padrão descrito para rios neotropicais, com a predominância de Characiformes e Siluriformes (Lowe McConnell, 1999). Porém, após o barramento houve uma inversão quanto ao número de espécies dessas ordens. Na Etapa I, a ordem Siluriformes apresentava maior representatividade e após o barramento (Etapas II, III e IV) a ordem Characiformes apresentou maior representatividade. A maior alteração nestas ordens, porém, está na abundância, onde, após a implantação da PCH constatou-se um grande aumento na abundância de Characiformes e um grande decréscimo na abundância de Siluriformes. Estas alterações podem estar relacionadas a formação de um novo ambiente ocasionado pelo barramento do rio que, segundo Agostinho et al. (2007), leva à criação de novos hábitats e perda de outros. Dentre os hábitats novos, cabe ressaltar a zona pelágica (Agostinho et al., 2007), onde são encontrados principalmente os representantes da família Characidae, cujos membros geralmente formam o principal conjunto de espécies de meia água (Buckup, 1999). Dentre os hábitats perdidos, destacam-se as corredeiras (Agostinho et al., 2007), onde são encontradas diversas espécies da família Loricariidae, características deste tipo de hábitat (Buckup, 1999). Assim, no presente estudo, a perda das corredeiras pode ter resultado na diminuição da abundância de Siluriformes e o acréscimo da zona pelágica pode ter resultado no aumento da abundância de Characiformes.

Conforme Matthews (1998), na maioria das comunidades animais, há poucas espécies abundantes e muitas espécies representadas por poucos indivíduos. Esta afirmação se confirmou nos resultados do presente estudo durante as quatro etapas, porém constatou-se que as espécies mais abundantes não foram sempre as mesmas. As abundâncias mais elevadas ficaram restritas principalmente a Astyanax spp., H. punctulatus, S. biornata, O. jenynsi, G. brasiliensis e H. luetkenii.

As análises estatísticas utilizadas, como ANOVA, podem não estar revelando as reais modificações sofridas pela comunidade. Tais análises baseiam-se em dados quantitativos, sendo altamente influenciadas por espécies com altas taxas de abundância e pouco influenciadas por espécies pouco abundantes. Assim, o desaparecimento de espécies raras e a alteração das espécies mais abundantes nas diferentes etapas não são refletidas.
Uma explicação para a não significância estatística da ANOVA também entre montante e jusante ao longo das etapas, pode ser o fato de que o trecho a jusante do barramento também teve sua característica lótica alterada devido à diminuição da vazão ocasionada pelo barramento. Além disso, existe um obstáculo natural (uma queda d'água de aproximadamente três metros de altura) localizado antes da casa de máquinas, que em virtude da diminuição do fluxo de água, geralmente encontra-se seco. Assim, entre a barragem e a casa de máquinas, forma-se um trecho de rio com pouco fluxo e relativamente isolado.

A transformação das águas lóticas de um rio em lênticas ou semilênticas de um reservatório tem efeitos adversos sobre a biota. Espécies que não encontrarem as condições adequadas para satisfazerem suas demandas ecológicas no novo ambiente poderão ter a sua abundância reduzida, ou mesmo serem eliminadas (Agostinho, 1992). No presente estudo, verificou-se o desaparecimento de nove espécies após o barramento, das quais sete já possuíam ocorrência acidental na área de implantação. Assim, é provável que as modificações advindas da implantação da PCH tenham exercido um maior impacto sobre as espécies consideradas acessórias e acidentais e/ou menos abundantes, causando uma diminuição na riqueza local de espécies.

$\mathrm{O}$ aumento repentino da quantidade de água e a elevada disponibilidade de alimento no início da formação do reservatório, especialmente os de origem alóctone (Petrere JR. \& Ribeiro, 1994; Agostinho \& Júlio Jr., 1999), pode explicar o aumento da diversidade a montante e sua diferença significativa com relação a jusante do barramento na Etapa II. Porém, esta disponibilidade de alimento tende a decrescer após os primeiros anos da formação do reservatório (PETRERE JR. \& RIBEIRO, 1994), promovendo novas alterações populacionais que refletem na estrutura da comunidade (AgostinHo et al., 2003). As alterações na diversidade e abundância relativa das espécies ao longo das etapas podem estar associadas a um período de adaptação e restabelecimento frente ao novo ambiente. Resultados semelhantes podem ser observados com relação à equitabilidade. Já com a riqueza de espécies, pode-se observar uma redução após o barramento devido à diminuição no número de espécies, de 28 espécies registradas na Etapa I para 18 na Etapa IV, representando uma queda de $35,71 \%$.

Além da diminuição do número de espécies, algumas espécies tiveram sua abundância e constância de ocorrência reduzidas. Entre estas espécies destacamse Hemiancistrus punctulatus, Rineloricaria cadeae, Glanidium melanopterum e Crenicichla punctata. No caso de $H$. punctulatus, que tem como ambiente preferencial corredeiras e substrato rochoso (AGOSTINHO et al., 2003), houve uma grande redução na sua abundância ao longo do período de estudo, ainda que tenha se apresentado de forma relativamente constante nas Etapas II e III, passando à acessória na Etapa IV. Da mesma forma, constatou-se a redução na abundância e na constância de ocorrência nas amostragens para as demais espécies da família Loricariidae. Exceção pode ser feita à espécie $H$. commersoni, que manteve sua abundância e constância de ocorrência estáveis durante as quatro etapas do monitoramento. Esta espécie 
aparentemente possui a capacidade de desenvolver seu ciclo reprodutivo inteiramente em ambiente lêntico, como encontrado por AgostinHo et al. (1991) no reservatório de Capivari-Cachoeira. Assim, H. commersoni provavelmente esteja mais apta a adaptar-se às modificações causadas pelo barramento do que outras espécies de Loricariidae.

Por outro lado, outras espécies tiveram sua abundância e constância de ocorrência elevadas após o barramento do rio. Entre as espécies que apresentaram maiores aumentos estão A. jacuhiensis, $H$. luetkenii, $O$. jenynsii, S. biornata e G. brasiliensis. A proliferação de pequenos caracídeos tem sido observada em vários reservatórios (AgOSTINHO \& Júlio JR., 1999). No caso de O. jenynsii, é conhecida sua preferência por águas lênticas (Froese \& PAUly, 2005), o que poderia explicar o aumento em sua abundância. Também é freqüentemente observada a proliferação de G. brasiliensis em ambientes lênticos, como em vários reservatórios localizados no Estado de São Paulo (CAStro \& Arcifa, 1987), o que evidencia que os requerimentos ecológicos desta espécie a predispõem ao sucesso na colonização deste tipo de ambiente (ABELHA \& GoulART, 2004).

O efeito da alteração do fluxo hidrológico sobre o detrito e a capacidade dos detritívoros em utilizá-lo como recurso alimentar (Bowen, 1984), constitui uma possível justificativa para o aumento da abundância da espécie $S$. biornata e a permanência da espécie $H$. commersoni.

Hoplias malabaricus (Bloch, 1794), por ser espécie de ambiente lêntico (JúLIO JR. et al., 1997), poderia apresentar proliferação após o barramento, porém apenas apresentou um aumento sutil em sua abundância chegando a representar 2,26\% na Etapa IV. Apesar de o novo ambiente ser aparentemente favorável para a sua proliferação, flutuações bruscas no nível da água durante o período reprodutivo podem comprometer o sucesso reprodutivo dessa espécie (Suzuki \& Agostinho, 1997). Tais flutuações de nível da água foram comumente observadas no reservatório durante o monitoramento pós barramento.

Apesar de não amostrada durante a Etapa IV com a metodologia utilizada, D. speculiferum pode ser freqüentemente observada por visualização e coletas com puçá nas margens do reservatório, sugerindo que a abundância desta espécie é, provavelmente, muito maior do que a detectada.

Tal como citado anteriormente, as espécies mais atingidas devido às modificações ambientais impostas pelos reservatórios seriam as reofílicas (AgOstinHo, 1992). A única espécie reofílica registrada durante o período de estudo foi $P$. maculatus e a mesma apresentou baixa abundância e constância de ocorrência acidental. No estudo realizado no rio das Antas, Agostinho et al. (2003) encontraram cinco espécies reofílicas, número baixo se comparado às grandes bacias brasileiras. Dessas cinco espécies, $P$. maculatus foi a que apresentou distribuição mais ampla na região amostrada, sendo que as demais espécies ficaram restritas a um determinado trecho. Semelhantemente ao estudo de Agostinho et al. (2003), a localização da área estudada em relação à bacia do JacuíTaquari-Antas pode estar relacionada à baixa ocorrência de espécies reofílicas.
Outra indicação de alteração biológica no rio Forqueta pode ser constatada através da análise de cluster apresentada no dendograma da figura 4, onde nota-se uma diminuição na similaridade entre os trechos montante e jusante ao longo das quatro etapas. A alta similaridade entre os trechos na Etapa I é justificada pelo rio ainda não encontrar-se bloqueado pelo barramento, apresentando um fluxo contínuo de água. A partir da Etapa II, o barramento encontrava-se concluído e a $\mathrm{PCH}$ em funcionamento. O represamento reduziu consideravelmente o fluxo e o volume de água a jusante para uma vazão mínima de $0,24 \mathrm{~m}^{3} / \mathrm{s}$, sendo que a média da vazão na etapa anterior ao barramento era de $18 \mathrm{~m}^{3} / \mathrm{s}$. Sugere-se que estas condições ambientais ocasionadas pelo empreendimento, foram responsáveis pela redução da similaridade entre os trechos jusante e montante, como também entre as etapas ao longo do estudo.

Os resultados ora apresentados representam um dos primeiros esforços na compreensão das alterações na composição da comunidade de peixes em um rio do Rio Grande do Sul pela instalação de uma central hidrelétrica. Este estudo mostra uma avaliação inicial dos efeitos da instalação e funcionamento da PCH Salto Forqueta, sendo o primeiro levantamento da ictiofauna deste rio. Estudos futuros poderão revelar com mais precisão o real impacto deste empreendimento, visto que a comunidade ainda encontra-se em adaptação ao novo ambiente. Não obstante, seis espécies registradas neste trabalho, apresentam status taxonômico parcialmente indeterminado, isto é, que só foram identificadas até o nível de gênero, denotando o parco conhecimento da ictiofauna regional.

Agradecimentos. Agradecemos ao Dr. Luiz R. Malabarba e ao Msc. Juan A. Anza, do Laboratório de Ictiologia/ UFRGS, e ao Dr. Roberto E. Reis, do Laboratório de Ictiologia MCP/PUCRS, pelo auxílio na determinação das espécies. À $\mathrm{Dr}^{\mathrm{a}}$. Clarice B. Fialho, do Laboratório. de Ictiologia/ UFRGS, ao Dr. Fábio F. Lopes, da UESC/BA, e ao doutorando Carlos Benhur Kasper, do Departamento de Zoologia/UFRGS, pela revisão do presente artigo. Ao Dr. Eduardo Périco, da UNIVATES, pelo auxílio nas análises estatísticas e a todos os colegas da UNIVATES que auxiliaram em campo. Este estudo recebeu apoio financeiro da UNIVATES e da CERTEL.

\section{REFERÊNCIAS BIBLIOGRÁFICAS}

Abelha, M. C. F. \& Goulart, E. 2004. Oportunismo trófico de Geophagus brasiliensis (Quoy \& Gaimard, 1824) (Osteichthyes, Cichlidae) no reservatório de Capivari, Estado do Paraná, Brasil. Acta Scientiarum. Biological Sciences 26(1):37-45.

Agostinho, A. A. 1992. Manejo de recursos pesqueiros em reservatórios. In: Agostinho, A. A.; Benedito-Cecilio, E. \& Isancnahum. eds. Situação Atual e Perspectivas da Ictiologia do Brasil. Maringá, EDUEM. p.106-121.

Agostinho, A. A. \& Júlio JR., H. F. 1999. Peixes da bacia do alto rio Paraná. In: Lowe Mc ConNel, R. H. ed. Estudos ecológicos de comunidades de peixes tropicais. São Paulo, EDUSP. p.374-400.

Agostinho, A. A.; Gomes, L. C. \& Pelicice, F. M. 2007. Ecologia e manejo de recursos pesqueiros em reservatórios do Brasil. Maringá, EDUEM. 501p.

Agostinho, A. A.; Hahn, N. S. \& Agostinho, C. S. 1991. Ciclo reprodutivo e primeira maturação de fêmeas de Hypostomus commersoni (Valenciennes, 1840) (Siluriformes, Loricariidae) no reservatório Capivara-Cachoeira, PR. Revista Brasileira de Biologia 51(1):31-37.

Agostinho, A. A.; Latini, J. D.; Luz, K. D. G. Da \& Gomes, L. C. 
2003 A ictiofauna do Rio das Antas, área de influência do Complexo Energético Rio das Antas. Maringá, CERAN, LIMNOBIOS. 213p

Barrella, W.; Petrere Jr., M.; Smith, W. S. \& Montag, L. F. DE. A. 2000. As relações entre as matas ciliares, os rios e os peixes. In: Rodrigues, R. R. \& Leitão Filho, H. DE. F. eds. Matas ciliares: conservação e recuperação. São Paulo, EDUSP FAPESP. 320p.

Bowen, S. H. 1984. Detritivory in neotropical fish communities. In: ZARET, T. M. ed. Evolutionary ecology of neotropical freshwater fishes. Illinois, Dr. W. Junk. p.59-66.

Buckup, P. A. 1999. Sistemática e biogeografia de peixes de riacho. In: Caramaschi, E. P.; Mazzoni, R. \& Peres-Neto, P. R. eds. Ecologia de Peixes de Riachos. Série Oecologia Brasiliensis, Rio de Janeiro, PPGE-UFRJ. v.VI, p.91-138.

Castro, R. M. C. \& Arcifa, M. S. 1987. Comunidades de peixes de reservatório do sul do Brasil. Revista Brasileira de Biologia 47(4):493-500.

Dajoz, R. 1983. Ecologia Geral. Petrópolis, VOZES. 4 ed. $472 \mathrm{p}$.

Froese, R. \& Pauly. D. 2005. FishBase. World Wide Web electronic publication. Disponível em: <http:// www.fishbase.org>. Acesso em: 19.11.2005.

Jasper, A.; Freitas, E.; Musskopf, M. E. \& Bruxel, J. 2005. Metodologia de salvamento de Bromeliaceae, Cactaceae Orchidaceae na Pequena Central Hidrelétrica (PCH) Salto Forqueta - São José do Herval / Putinga - RS - Brasil. Pesquisas, Botânica 56:265-284.

Júlio JR., H. F.; Bonecker, C. C. \& Agostinho, A. A. 1997. Reservatório de Segredo e sua inserção na bacia do rio Iguaçu. In: Agostinho, A. A. \& Gomes, L. C. eds. Reservatório de Segredo: bases ecológicas para o manejo. Maringá, EDUEM. 387p.

Koch, W. R.; Milani, P. C. \& Grosser, K. M. 2000. Guia Ilustrado: peixes Parque Delta do Jacuí. Porto Alegre, Fundação Zoobotânica do Rio Grande do Sul. 91p.
Lowe McConnell, R. H. 1999. Estudos ecológicos de comunidades de peixes tropicais. São Paulo, EDUSP $535 \mathrm{p}$.

Malabarba, L. R. 1989. Histórico sistemático e lista comentada das espécies de peixes de água doce do sistema da Laguna dos Patos, Rio Grande do Sul, Brasil. Comunicações do Museu de Ciências e Tecnologia da PUCRS, Série Zoologia 2(8): 107-179.

Matthews, W. J. 1998. Patterns in Freshwater Fish Ecology. New York, Chapman \& Hall. 756p.

Nakatani, K.; Agostinho, A. A.; Baumgartner, G.; Bialetzki, A. Sanches, P. V.; Makrakis, M. C. \& Pavanelli, C. S. 2001. Ovos e Larvas de peixes de água doce: desenvolvimento e manual de identificação. Maringá, EDUEM. 378p.

Petrere JR., M. \& Ribeiro, M. C. L. B. 1994. The impact of large tropical hydroeletric dam: the case of Tucurui in the middle river Tocantins. Acta Limnologica Brasiliensia 5:123-133.

Reis, R. E.; Kullander, S. O. \& Ferraris Jr., C. J. 2003. Check list of the freshwater fishes of South and Central America. Porto Alegre, EDIPUCRS. 742p.

Schindler, D. W. 1987. Detecting ecosystem responses to anthropogenic stress. Canadian Journal of Fisheries and Aquatic Sciences 44(1):6-25

Suzuki , H. I. \& Agostinho, A. A. 1997. Reprodução de peixes do reservatório de Segredo. In: Agostinho, A. A. \& Gomes, L. C. eds. Reservatório de Segredo: bases ecológicas para o manejo. Maringá, EDUEM. 387p.

Teixeira, M. B.; Coura Neto, A. B.; Pastore, U. \& Rangel Filho, A L. R. 1986. Vegetação. As regiões fitoecológicas, sua natureza e seus recursos econômicos. Estudo fitogeográfico. In Levantamento de recursos naturais. Rio de Janeiro, Instituto Brasileiro de Geografia e Estatística. v.33, p.541-632.

Tundisi, J. G. 1999. Reservatórios como sistemas complexos: teoria, aplicações e perspectivas para usos múltiplos. In: Henry, R. ed. Ecologia de Reservatórios: estrutura, função e aspectos sociais. São Paulo, FUNDIBIO/ FAPESP. 800p.

Recebido em julho de 2007. Aceito em julho de 2008. ISSN 0073-4721

Artigo disponível em: www.scielo.br/isz 